\title{
Über die Verwertung intravenös zugeführter Eiweißabbauprodukte im Stoffwechsel.
}

I. Mitteilung.

\author{
Von \\ C. Oehme.
}

(Aus der medizinischen Klinik zu Göttingen.)

(Der Redaktion zugegangen am 21. Januar 1914.)

Die Mitteilung von Henriques und Andersen über parenterale Ernährung ${ }^{1}$ ) veranlaßt mich, über Versuche zu berichten, die vor fast einem Jahre begonnen wurden, aber aus äußern Gründen noch nicht abgeschlossen werden konnten. Sie gelten der bereits viel erörterten Frage, ob die im Darm durch die Verdauungsfermente gebildeten Spaltstücke der Eiweißkörper als solche im Stoff wechsel verwendet werden können oder ob ihre physiologische Brauchbarkeit abhängig ist von einer mehr oder weniger weitgehenden Resynthese in der Darmwand oder in der Leber.

Von Abderhalden u. a. sind Aminosäuren im Blute nachgewiesen worden; die Untersuchungen Folins u. a. haben gezeigt, daß während der Resorption von Aminosäuren der Reststickstoff (abzüglich des Harnstoffstickstoffs) im Blute ansteigt. Es ist nicht der Ort, in dieser vorläufigen Mitteilung des näheren auf die zahlreichen Forschungen in dieser Richtung einzugehen, welche zu einem Abschluß der Frage noch nicht geführt haben.

Der endgültige Beweis dafür, daß die Eiweißspaltstücke keinem Wiederaufbau in der Darmwand zu unterliegen brauchen,

1) S. diese Zeitschrift, Bd. 88, S. 5 . 
um dem Proteinmolekül im Stoffwechsel gleichwertig zu sein, würde erst dann als erbracht gelten können, wenn es gelänge, die durch Fütterungsversuche bekannten Gesetze des N-Stoffwechsels (N-Gleichgewicht, N-Minimum usw.) durch intravenöse Zufuhr des $\mathbf{N}$ in Gestalt eines alle Komponenten des ursprünglichen Eiweißkörpers enthaltenden Gemisches von Spaltstücken zu reproduzieren. Im besonderen galt meine Aufmerksamkeit zunächst der Frage, ob es möglich ist, den Körperstickstoff, der im Hunger oder bei $\mathrm{N}$-armer Kohlenhydratfettkost zur Ausscheidung gelangt, durch intravenöse Zufuhr der Eiweißabbauprodukte ganz oder teilweise vor dem Zerfall zu schützen.

Die Schwierigkeit solcher Versuche liegt vor allem darin, daß bei dem fermentativen Abbau von Eiweiß in vitro, der zu diesem Zwecke möglichst weitgehend anzustreben ist, sich das Auftreten proteinogener Amine bis heute meines Wissens nicht vermeiden läßt, wahrscheinlich weil trotz Antisepticis bakterielle Umsetzungen in lange stehenden Verdauungsgemischen nie ganz ausbleiben. Diese Stoffe (wie Histamin u. a.) und auch andere bei der Spaltung frei werdende Körper (z. B. Tryptophan) entfalten aber, intravenös injiziert, eine starke Giftwirkung. So zeigen z. B. Kaninchen (Gew. 1800, resp. 2100 g), denen 4,5 resp. $5,5 \mathrm{ccm}$ einer 6\% igen Ereptonlösung ${ }^{1}$ ) intravenös schnell injiziert werden, schwere Vergiftungssymptome (Krämpfe, Dyspnoë), die unter Atemstillstand sofort zum Tode führen. Diese Giftwirkung bleibt aus, wenn die intravenöse Zufuhr langsam erfolgt. Das giftige Prinzip des Ereptons (aller Wahrscheinlichkeit nach mehrere verschiedene Körper) gleicht hierin dem Histamin und andern Giften vom Typus der Potentialgifte.

Auf Grund dieses Ergebnisses habe ich begonnen zu untersuchen, ob der im Hunger oder bei $\mathrm{N}$-armer Kohlenhydratfettkost zerfallende Körperstickstoff bei langsamer intravenöser Zufuhr durch Eiweißabbauprodukte vertretbar ist. Benutzt wurde vorläufig nur das im Handel befindliche Erepton.

Da der Grad der Spaltung bei den einzelnen Ereptonpräparaten wechselt, wurde in jedem Falle die injizierte Flüssigkeit analysiert (s. Tab. A).

i) Nach Abderhaldens Angaben abgebautes Muskelfleisch, von Meister, Luçius und Brüning dargestellt. 


\section{Tabelle A.}

Analysen der in Versuch I-IV injizierten Ereptonlösungen. Auf $100 \mathrm{~g}$ Erepton-N (Total) entfallen:

\begin{tabular}{c|r|c|c|c|c}
\hline InVersuch & $\mathrm{NH}_{3}$ & $\mathrm{NH}_{2}$ & $\begin{array}{c}\text { Nicht formol- } \\
\text { titrierbarer N }\end{array}$ & $\begin{array}{c}\text { Davon } \\
\text { Peptid-N }\end{array}$ & $\mathrm{C}$ \\
\hline I & 11,15 & 61,3 & 27,55 & 15,2 & 346 \\
II & 9,85 & 57,2 & 32,95 & - & 346 \\
III & 10,55 & 74,8 & 9,7 & - & - \\
IV & 11,41 & 63,6 & 24,99 & - & -
\end{tabular}

Im Mittel entfallen auf 100 Teile Erepton-N ca. 75\% formoltitrierbarer $\mathrm{N}$, wovon $10 \%$ als $\mathrm{NH}_{3}-\mathrm{N}$ in Rechnung zu setzen sind. Unter den ca. $25 \%$ nicht formoltitrierbarem $\mathrm{N}$ befinden sich ca. $15 \%$ Peptid-N, die nach 3 stündiger Hydrolyse mit gleichen Teilen $50 \% \mathrm{HCl}$ mit Hilfe der Formoltitration nachweisbar werden. Dieser Wert für Peptid-N ist, nach Maßgabe der Methodik, natürlich nur als unterer Grenzwert zu betrachten. N-Bestimmung erfolgte nach $\mathrm{Kjeldahl,} \mathrm{NH}_{3}$-Best. nach $\mathrm{Kr}$ ügerReich-Schittenhelm, $\mathrm{NH}_{2}$-Best. und teilweise Peptid-Best. nach Sörensen, C-Best. auf feuchtem Wege (Veraschung mit Kaliumbichromat und Schwefelsäure) nach Messinger. Letztere wurde nur in den beiden ersten Versuchen in der Absicht vorgenommen, aus dem bei gleichmäßiger Kost beim Hund so konstanten Quotienten $\frac{\mathrm{C}}{\mathrm{N}}$ eventuell einen Rückschluß auf die Form zu ziehen, in der der injizierte $\mathrm{N}$ ausgeschieden wurde. Bei allen Versuchen wurde für Körperwärme der einfließenden Flüssigkeit Sorge getragen.

Eine Vorfrage war, ob von dem intravenös zugeführten Aminosäuregemisch ein mehr oder weniger großer Teil im Harn als formoltitrierbarer $\mathrm{N}$ ausgeschieden wird und inwieweit hierauf etwa die Ernährungsweise von Einfluß ist. Versuche, die das Schicksal intravenös eingespritzter Eiweißabbauprodukte verfolgen und die mir zu Beginn meiner Arbeit nicht bekannt waren, hat bereits $B_{\text {uglia }}{ }^{1}$ ) unternommen und gefunden, daß ein großer Teil des Aminosäure- $\mathrm{N}$ als Harnstoff- $\mathrm{N}$ erscheint. Folgende zwei Versuche bestätigen dieses Resultat:

Versuch I ist an einem Hungertier vorgenommen, während bei II der Hund eine $\mathrm{N}$-arme Nahrung erhielt. Nachdem die Vena jugularis

1) Zeitschr. f. Biologie, Bd. 58. 
unter Novocain-Adrenalin-Lokalanästhesie freigelegt worden war, wurden aus einer Bürette innerhalb zweier Stunden $50 \mathrm{ccm}$ einer warmen Ereptonlösung einlaufen gelassen; die bei $\mathrm{I} 1,327 \mathrm{~g} \mathrm{~N}$ und $0,812 \mathrm{~g} \mathrm{NH_{2 }}-\mathrm{N}$, bei II $1,218 \mathrm{~g} \mathrm{~N}$ und $0,695 \mathrm{~g} \mathrm{NH}_{\mathbf{2}}-\mathrm{N}$ enthielt. Gerinnung in Vene oder Kanüle wurde weder in diesen noch in den folgenden Experimenten beobachtet, offenbar infolge Gegenwart gerinnungshemmender Substanzen im Erepton. Überschreitet man mit der Injektionsgeschwindigkeit oder der Konzentration der Lösung eine gewisse Grenze, so tritt als erste toxische Wirkung Erbrechen und Entleerung eines oft diarrhöischen Kotes ein, wie Fehlversuche lehrten. Diese Beeinflussung des Magendarmkanals muß vermieden werden, weil sie an sich eine Veränderung des zu beobachtenden N-Umsatzes hervorrufen kann und weil mit der Möglichkeit zu rechnen ist, daß im Erbrochenen oder im flüssigen Kote teilweise die injizierten Substanzen ausgeschieden werden. In den vorliegenden Versuchen wurde der Kot nur alle 2-3 Tage, nie im Anschluß an die Injektion, in konsistenter Form entleert, sodaß eine Ausscheidung von injiziertem $N$ auf diesem Wege ausgeschlossen sein dürfte. Hingegen gelang es nicht, in Versuch I Erbrechen einer geringen Menge Magenschleims, in Versuch IV von Speisebrei, der zwei Stunden vorher genossen worden war (s. u.), zu vermeiden.

Versuch I. - Tabelle I.

\begin{tabular}{|c|c|c|c|c|c|c|c|c|}
\hline Dat. & $\mathbf{N}$ & $\mathrm{NH}_{8}$ & $\mathrm{NH}_{2}$ & $\frac{\left(\mathrm{NH}_{3}\right) \mathrm{N}}{\mathrm{N}} \times 100$ & C & $\frac{\mathrm{C}}{\mathrm{N}}$ & $\begin{array}{l}\text { Urin- } \\
\text { menge }\end{array}$ & $\begin{array}{c}\text { Deutsche Dogge, } 8 \mathrm{~kg} . \\
\text { Hungert seit } 12 . / \mathrm{V} \text {. }\end{array}$ \\
\hline 14./V. & 3,085 & 0,279 & 0,0828 & 7,45 & - & - & 86 & Hunger \\
\hline 15. & 2,537 & 0,280 & 0,0588 & 9,06 & 2,0 & 0,788 & 75 & $\gg$ \\
\hline 16. & 2,07 & 0,266 & 0,0440 & 10,58 & 1,72 & 0,857 & ca. 90 & » \\
\hline 17. & 3,740 & 0,492 & $0, \mathbf{3 1 1} !$ & 10,8 & 5,38 & 1,440 & 560 & 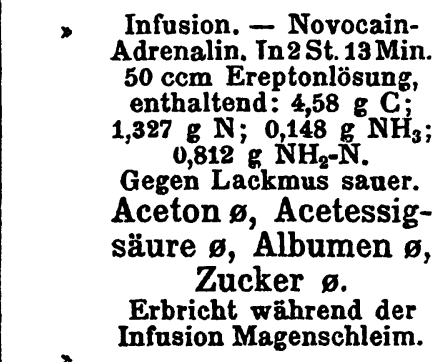 \\
\hline 18. & $3,5 \cup 0$ & $?>0,31$ & 0,0044 & $7,71 ?$ & 8,20 & 0,000 & 100 & 2 \\
\hline 19. & 2,45 & 0,250 & 0,0828 & 8,4 & 1,855 & 0,757 & 160 & • \\
\hline 20. & 2,385 & 0,240 . & 0,0520 & 8,25 & - & - & 110 & $>$ \\
\hline
\end{tabular}


Versuch II. - Tabelle II.

\begin{tabular}{|c|c|c|c|c|c|c|c|c|}
\hline Datum & $\mathbf{N}$ & $\mathrm{NH}_{3}$ & $\mathrm{NH}_{2}$ & $\frac{\left(\mathrm{NH}_{3}\right) \mathrm{N}}{\mathrm{N}} \times 100$ & C & $\frac{\mathrm{C}}{\mathrm{N}}$ & $\begin{array}{c}\text { Urin- } \\
\text { menge }\end{array}$ & 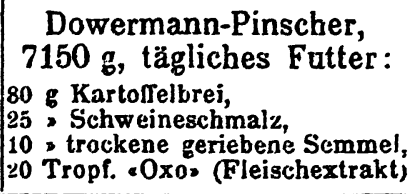 \\
\hline 12./III. & 1,34 & 0,0782 & 0,0301 & - & - & - & 80 & \\
\hline 13. & - & 0,0652 & 0,0663 & - & - & - & 95 & \\
\hline 14. & 1,277 & 0,0526 & 0,0441 & 4,13 & 1,122 & 0,878 & 120 & \\
\hline 15. & 1,249 & 0,0714 & 0,0421 & $\overline{5,72}$ & 1,077 & 0,861 & 115 & \\
\hline 16. & 1,351 & 0,0679 & 0,0442 & 4,14 & 1,064 & 0,787 & 108 & \\
\hline 17. & 2,335 & 0,0504 & $0,0780 !$ & 1,78 & 2,445 & 1,048 & $\begin{array}{l}\text { ca. } 220 \\
\text { kein } \\
\text { Ver- } \\
\text { lust }\end{array}$ & 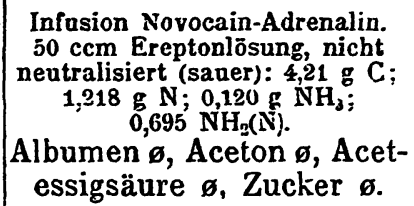 \\
\hline 18. & 1,850 & 0,0604 & 0,0328 & 2,69 & 1,574 & 0,851 & 200 & \\
\hline 19. & 1,403 & 0,0525 & - & 3,08 & 1,321 & 0,942 & 230 & \\
\hline
\end{tabular}

Bei dem Hungertier I übertrifft die $\mathrm{N}$-Ausscheidung im Harn die zugeführte N-Menge bedeutend. Wahrscheinlich ist dies wenigstens größenteils auf das Erbrechen während der Infusion zurückzuführen; bei II beträgt die Mehrausscheidung von $\mathrm{N}$ höchstens $0,3 \mathrm{~g}$. Im ersteren Falle erscheinen von $812 \mathrm{mg}$ injiziertem formoltitrierbarem $\mathrm{N}$ ca. $249 \mathrm{mg}$ (Ausscheidung am Injektionstag minus Mittel der Vorperiode), d. i. 29,55\%, im zweiten von $695 \mathrm{mg}$ nur ca. $35 \mathrm{mg}$, d. i. $5,03 \%$ als formoltitrierbarer $\mathrm{N}$ im Harn. Es liegt nahe, für diesen Unterschied in der Abspaltung des Amino- $\mathrm{N}$ die in beiden Versuchen weit auseinander liegende Größe der $\mathrm{NH}_{3}$-Bildung (gemessen an der Ausscheidung) verantwortlich zu machen, die bei I wohl infolge von Hungeracidose dazu geführt hat, den Quotienten $\frac{\left(\mathrm{NH}_{3}\right) \mathrm{N}}{\mathrm{N}} \times 100 \mathrm{im}$ Vergleich zu Versuch II auf das Doppelte zu erhöhen. Da aller Wahrscheinlichkeit nach der Vorgang der Desaminierung sowie der Harnstoff bildung ${ }^{1}$ ) einen Endzustand darstellt, wird durch die relativ hohe $\mathrm{NH}_{3}$-Konzentration bei Acidose die Abspaltung der Aminogruppen gehemmt.

1) Lichtwitz, Diese Zeitschrift, Bd. 77. 
Weder bei Hund I noch bei II ist der injizierte $\mathrm{N}$ nachweisbar für Körper-N eingetreten. Unter den gewählten Versuchsbedingungen (eine zweistündige Injektion) ist dies aber auch nicht zu erwarten. Aus den Untersuchungen von Thomas ${ }^{1}$ ) über die physiologische Wertigkeit der Eiweißkörper geht hervor, daß der bei kalorisch genügender Kohlenhydratfettkost zerfallende Körper- $N$ durch den Nahrungs- $N$ bestenfalls ( $d . h$. volle "Wertigkeit» des betreffenden Eiweißes vorausgesetzt) nur dann völlig substituiert werden kann, wenn die Eiweißgabe in kleinen Portionen über den ganzen Tag verteilt wird, sodaß sich der Organismus niemals im Zustande des N-Hungers befindet. Es ist also hiernach eine möglichst lange zeitliche Ausdehnung der intravenösen $\mathrm{N}-Z$ Zufuhr in unsern Versuchen anzustreben, und auch dann ist kein volles, sondern nur ein partielles Eintreten des Erepton- $N$ für den Körper-N zu erwarten, weil, wie gesagt, vorläufig in allen fermentativ gewonnenen Eiweißabbaugemischen sich aminartige Körper in geringer Menge vorfinden, deren physiologischche Brauchbarkeit im N-Stoffwechsel höchst zweifelhaft erscheint. Es ist wahrscheinlich, daß unter anderen sich gerade diese Substanzen unter den 5\% formoltitrierbaren $\mathrm{N}$ befinden, welcher auch im Versuch II trotz niedrigen $\frac{\left(\mathrm{NH}_{3}\right) \mathrm{N}}{\mathrm{N}} 100=$ Quotienten noch als solcher im Harn ausgeschieden worden ist. Hierzu kommt, daß möglicherweise auch die zur Infusion nötige langdauernde Fesselung des Hundes trotz erleichternder Vorsichtsmaßregeln Störungen im N-Umsatz hervorrufen kann, ein Punkt, über den weitere Untersuchungen angestellt werden. Die Isotonie und die Reaktion der zu injizierenden Flüssigkeit ist ebenfalls zu berücksichtigen, obwohl bei so außerordentlich langsamen Infusionen wie den vorliegenden kleinere Abweichungen von der Norm infolge der ausgleichenden Nierentätigkeit weniger in Frage kommen dürften. Nebenbei sei hier auf die diuretische Wirkung des Ereptons hingewiesen. Diesen Bedingungen wurde in den folgenden zwei Versuchen nach Möglichkeit Rechnung getragen und zugleich wurde, um einer Ausschwemmung von

1) Arch. f. Anat. u. Physiol. Physiol. Abt. 1909. 
Stickstoff durch die Flüssigkeitsmenge zuvorzukommen, den Tieren bereits in der Vorperiode täglich 200-300 ccm Wasser mit der Schlundsonde beigebracht. Im III. Versuch sollte gleichzeitig klargestellt werden, ob die bei dem Hungertier I beobachtete mangelhafte Desaminierung sich beheben läßt, wenn durch Alkaligaben die $\mathrm{NH}_{3}$-Bildung stark eingeschränkt wird.

Versuch 3. - Tabelle III.

\begin{tabular}{|c|c|c|c|c|c|c|c|}
\hline Datum & $\begin{array}{c}\text { Urin- } \\
\text { menge }\end{array}$ & $\mathrm{N}$ & $\mathrm{NH}_{3}$ & $\mathrm{NH}_{2}$ & $\begin{array}{c}\text { Peptid- } \\
\mathrm{N}\end{array}$ & $\frac{\left(\mathrm{NH}_{s}\right) \mathrm{N}}{\mathrm{N}} \times 100$ & Dogge, 20270 g. Hunger. \\
\hline 3. XI. & 335 & $\mid 5,84$ & 0,464 & 0,1103 & - & 6,53 & $\begin{array}{l}\text { Urin schwach sauer, hell, klar. } \\
\text { Spur Albumen-Trübung. Kein } \\
\text { Sediment renalen Ursprungs. }\end{array}$ \\
\hline 4. & 400 & 7,7 & 0,563 & 0,1413 & - & 6,01 & Hunger. \\
\hline 5. & $27 \check{5}$ & 7,48 & 0,425 & 0,1682 & - & 4,67 & $\begin{array}{l}\text { Desgl. } 40 \mathrm{~g} \mathrm{Na} \text { bicarb. + citric. } \overline{\text { aa }} \\
\text { in } 400 \mathrm{ccm} \mathrm{H}_{2} \mathrm{O} .1 / 2 \text { Stde. } \\
\text { später alles erbrochen. }\end{array}$ \\
\hline 6. & 450 & 6,19 & 0,110 & 0,1160 & 0,260 & 1,462 & $\begin{array}{l}10 \mathrm{~g} \mathrm{Na} \text { bicarb. }+ \text { citric. } \overline{\mathrm{aa}} \\
\text { in } 300 \mathrm{ccm} \mathrm{H}_{2} \mathrm{O} \text {. Kein Er- } \\
\text { brechen. }\end{array}$ \\
\hline 7. & 400 & 5,33 & 0,0929 & 0,1180 & 0,160 & 1,492 & $\begin{array}{l}\text { " } 15 \mathrm{~g} \mathrm{Na} \text { bicarb. + citric. in } \\
300 \mathrm{ccm} \mathrm{H}_{2} \mathrm{O} \text {. }\end{array}$ \\
\hline 8. & 535 & 6,01 & 0,127 & 0,1699 & 0,167 & 1,74 & $\begin{array}{l}12 \mathrm{~g} \mathrm{Na} \text { bicarb. + citric. in } \\
400 \mathrm{ccm} \mathrm{H}_{2} \mathrm{O} \text {. Acetessig- } \\
\text { säure }- \text { Aceton - }\end{array}$ \\
\hline 9. & 430 & 4,72 & 0,102 & 0,1398 & 一 & 1,78 & $\begin{array}{l}12 \mathrm{~g} \mathrm{Na} \text { bicarb. + citric. in } \\
400 \mathrm{ccm} \mathrm{H}_{2} \mathrm{O} \text {. }\end{array}$ \\
\hline $\begin{array}{l}10 \text { a. } \\
\text { während } \\
\text { der } \\
\text { Infusion }\end{array}$ & 190 & 3,28 & 0,0865 & 0,1760 & 0,323 & 2,175 & $\begin{array}{l}\text { Infusion: in } 9 \text { Std. } 7 \text { Min. } 448,05 \mathrm{ccm} \\
\text { Ereptonlösung mit } 2,13 \mathrm{~g} \mathrm{~N}, 0,239 \mathrm{~g} \mathrm{NH}_{3}, \\
1,592 \mathrm{~g} \mathrm{NH} \mathrm{NH}_{2} \mathrm{~N} . \Delta=-0,71 \text {. Neutralisiert } \\
\text { mit } \mathrm{NaHCO}_{3} \text { gegen Lackmus. } 2 \text { Stunden }\end{array}$ \\
\hline $\begin{array}{c}10 \mathrm{~b} . \\
\text { Rest }\end{array}$ & 790 & 4,34 & 0,12 & 0,1490 & - & 2,280 & $\begin{array}{l}\text { vor der Infusion: } 7 \mathrm{~g} \mathrm{Na} \text { bicarb. und } \\
\text { citric. } \mathrm{aa} \text { in } 200 \mathrm{ccm} \mathrm{H}_{2} \mathrm{O} \text {. Tier säuft }\end{array}$ \\
\hline Summe & 980 & 7,62 & 0,2065 & 0,325 & 一 & 2,23 & $\begin{array}{l}\text { gegen Ende d } \\
\text { Kein Erbrech }\end{array}$ \\
\hline 11. & 600 & 8,59 & 0,102 & 0,1180 & - & 0,976 & $\begin{array}{c}12 \mathrm{~g} \mathrm{Na} \text { bicarb. }+ \text { citric. } \overline{\mathrm{aa}} \text { in } \\
400 \mathrm{ccm} \mathrm{H}_{2} \mathrm{O} .\end{array}$ \\
\hline 12. & 330 & 6,48 & 一 & - & - & - & 16700 g Gewicht. \\
\hline
\end{tabular}

Injiziert wurden in 9 Stunden 7 Minuten 448,05 ccm einer ca. 4\% $\%$ igen Ereptonlösung, die mit $\mathrm{NaHCO}_{3}$ auf amphotere Reaktion gegen Lackmus gebracht war und einen Gefrierpunkt $\Delta=-0,71$ hatte, insgesamt 2,13 $\mathrm{g} \mathrm{N}, 0,239 \mathrm{~g} \mathrm{NH}_{3}, 1,592 \mathrm{~g}$ formoltitrierbarer $\mathrm{N}$. In der Vorperiode waren dem Hund täglich $10-15 \mathrm{~g} \mathrm{Na}$ bicarb. + citric. $\overline{\mathrm{aa}}$ in $300 \mathrm{H}_{2} \mathrm{O}$ gegeben worden. Die Infusion verlief ohne irgend welche Störungen. 
Über intravenöse Eiweißabbauprodukte im Stoffwechsel. I. 319

Es gelang in diesem Versuche nicht, Stickstoffgleichgewicht in der Vorperiode $z u$ erreichen, wohl infolge der wechselnden Größe der Salzresorption. Deshalb läßt sich ein eventuelles Einsparen von Körper- $\mathrm{N}$ durch den Erepton- $\mathrm{N}$ hier nicht berechnen. Hingegen ist deutlich zu ersehen, daß bei einem infolge der Alkaliwirkung sehr niedrigen Quotienten $\frac{\left(\mathrm{NH}_{3}\right) \mathrm{N}}{\mathrm{N}} \times 100$ die Desamidierung viel weiter geht als bei dem Hungertier I. Von $1592 \mathrm{mg}$ formoltitrierbarem $\mathrm{N}$ erscheinen $187,9 \mathrm{mg}$, d. i. $11,79 \%$ (gegen $29 \%$ bei I) als solche im Harn. Obwohl die relative $\mathrm{NH}_{3}$-Ausscheidung der Vorperiode hier noch geringer ist als bei Versuch II $\left(\frac{\left(\mathrm{NH}_{3}\right) \mathrm{N}}{\mathrm{N}} 100=\right.$ ca. 1,5 gegen $4-5$ bei II), wird doch mehr Amino- $N$ mit dem Harn eliminiert als bei Kohlenhydratfettkost, sodaß yermutet werden kann, es möchten noch andere Faktoren als die $\mathrm{NH}_{3}$-Größe eine gewisse Rolle bei der Desaminierung spielen. Daß dies in der Tat der Fall ist, erhellt beșonders aus Versuch IV.

Versuch IV. - Tabelle IV.

\begin{tabular}{|c|c|c|c|c|c|c|}
\hline Datum & $\begin{array}{c}\text { Urin- } \\
\text { menge }\end{array} \mid$ & $\mathbf{N}$ & $\mathrm{NH}_{3}$ & $\mathrm{NH}_{2}$ & $\frac{\left(\mathrm{NH}_{3}\right) \mathbf{N}}{\mathrm{N}} \times 100$ & $\begin{array}{l}\text { Kleiner Pinscher, } 4200 \mathrm{gg} . \\
\text { Voraufgehend } 2 \text { Tage Hunger, vom } 13 \text {. XII. an } \\
\text { tägl. Futter: } 10 \mathrm{~g} \text { trockene, zerriebene Semmel. } \\
80 \mathrm{~g} \text { Schweineschmalz. } 70 \mathrm{~g} \text { Kartoffeln. }\end{array}$ \\
\hline 13./XII. & $\left|\begin{array}{c}? \\
\text { kein } \\
\text { Verlust }\end{array}\right|$ & 1,664 & 一 & - & - & $\begin{array}{c}\text { Urin hellgelb, schwach sauer, klar. } \\
\text { Albumosen - }\end{array}$ \\
\hline 14. & 130 & 1,24 & - & - & - & $\begin{array}{l}\text { Frißt täglich quantitativ sofort bei Futter- } \\
\text { darreichung. } \\
300 \mathrm{ccm} \mathrm{H}_{2} \mathrm{O} \text {, warm, mit Sonde. Erbricht bald } \\
\text { darnach } 300 \mathrm{ccm} .\end{array}$ \\
\hline 15. & 350 & 1,123 & 0,133 & 0,0230 & 9,69 & $250 \mathrm{ccm} \mathrm{H}_{2} \mathrm{O}$ mit Sonde. \\
\hline 16. & 450 & 1,090 & 0,1632 & $0,02: 35$ & 12,34 & 250 \\
\hline 17. & 450 & 1,131 & 0,1079 & 0,0217 & 7,85 & Frißt $36 \mathrm{~g}$ des Gemisches nicht. \\
\hline 18. & 850 & 2,015 & 0,1274 & 0,0503 & 5,2 & 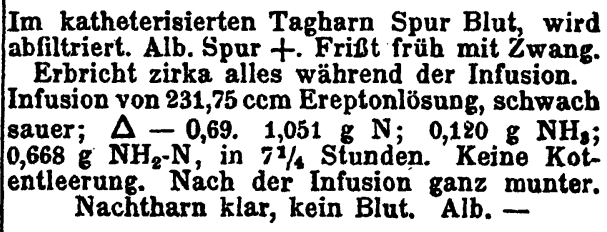 \\
\hline 19. & 200 & 2,15 & 0,1413 & 0,0195 & 5,41 & $\begin{array}{l}\text { Futter wie oben, nur } 50 \mathrm{~g} \text { Schweineschmalz, } \\
\text { Fribt das Gemisch bis auf } 10 \mathrm{~g} \text {. }\end{array}$ \\
\hline 20. & $\left|\begin{array}{c}? \\
\text { kein } \\
\text { Verlust }\end{array}\right|$ & 1,945 & - & - & - & Futter quantitativ gefressen. \\
\hline
\end{tabular}

Hoppe-Seyler's Zeitschrift f. physiol. Chemie. LXXXIX. 
Hier wurde zu der Kohlenhydratfettkost wie im 2. Versuche zurückgekehrt; nur die Infusionsdauer auf $71 / 4$ Stunden ausgedehnt und in dieser Zeit $231,75 \mathrm{ccm}$ einer Ereptonlösung injiziert, die $1,051 \mathrm{~g} \mathrm{~N}, 0,120 \mathrm{~g} \mathrm{NH}_{3}$ und $0,0668 \mathrm{~g}$ formoltitrierbaren $\mathrm{N}$ bei einem Gefrierpunkte von $\Delta=-0,69$ enthielt. Hier liegt trotz der gleichen Diät wie bei II der Quotient, $\frac{\left(\mathrm{NH}_{3}\right) \mathrm{N}}{\mathrm{N}} \times 100$ wesentlich höher, und dennoch wurden von $668 \mathrm{mg}$ $\mathrm{NH}_{2}-\mathrm{N}$ im Mittel nur 27,6 mg, d. i. 4,13\% im Harn ausgeschieden, soda $B$ auf eine prompte Desamidierung geschlossen werden darf. Es sei nur kurz bemerkt, daß bei den Hungerversuchen I und III die $\mathrm{NH}_{3}$-Ausscheidung an den Infusionstagen stets beträchtlich ansteigt, während bei Kohlenhydratfettkost (II und IV) die absolute $\mathrm{NH}_{2}$-Größe im Harn sich trotz des $\mathrm{NH}_{3}$-Gehalts der injizierten Ereptonlösung kaum ändert. Vielleicht weist auch dies darauf hin, daß bezüglich des Vorganges der Desaminierung nicht allein die relative Größe der Ammoniakausscheidung, sondern auch die spezielle Verwendung desselben im Stoffwechsel bei reichlicher Anwesenheit von Kohlenhydraten und Fettsäuren von Wichtigkeit ist.

In Versuch IV bleibt die N-Ausscheidung am Injektionstage etwas hinter der zugeführten Menge zurück, aber noch an 2 Tagen der Nachperiode liegt sie $0,9-1,0 \mathrm{~g}$ über dem Mittelwert der Vortage. Während am Injektionstage die durch Erbrechen bedingte ungenügende Nahrungsaufnahme mindestens teilweise als die Ursache des $\mathrm{N}$-Anstieges im Harn anzusehen ist, gewinnt man aus den höheren N-Zahlen der Nachperiode den Eindruck, daß die Injektion von Erepton eine länger dauernde Störung (Reizwirkung) des N-Stoffwechsels herbeigeführt hat.

Aus diesen vorläufigen Versuchen ergibt sich also, daß der Stickstoff intravenös injizierter Eiweißabbauprodukte in weitgehender Weise im Stoffwechsel verarbeitet wird. Es ist aber bisher nicht gelungen, Körperstickstoff dadurch vor Zerfall zu schützen. Damit ist jedoch die Frage nicht erledigt, ob dies überhaupt prinzipiell unerreichbar sei. Denn, wie erörtert, sind die experimentellen Anforderungen, welche erfüllt werden müssen - Vollwertigkeit des Abbaugemisches, Ab- 
wesenheit bakterieller Zersetzungsprodukte, möglichst gleichmäßige, über den Tag verteilte Zufuhr usw. - so zahlreich und z. T. so kompliziert, daß ihre exakte Erfüllung noch in weiteren Versuchen anzustreben ist.

Von den Versuchen von Henriques und Anderson unterscheiden sich die meinen erstens in der Fragestellung, insofern als jene N-Gleichgewicht in längerer Versuchsperiode erzielen wollten und tatsächlich erreichten; sodann aber besonders insofern, als jene gleichzeitig große Mengen von Zucker und Na-Acetat intravenös zuführten. Diese Versuchsanordnung weicht aber von den natürlichen, im Darm gegebenen Resorptionsbedingungen, deren Reproduktion auf intravenösem Wege von mir erstrebt wurde, weit ab. Es bleibt zu fragen, ob das auf diese Weise erzielte N-Gleichgewicht physiologisch tatsächlich dem gleichzusetzen ist, welches wir aus den Fütterungsexperimenten kennen, oder ob bei Gegenwart großer Mengen von Na-Acetat und Zucker Stickstoff in anderer als der gewöhnlichen Form zurückgehalten und so ein N-Gleichgewicht eventuell vorgetäuscht werden kann.

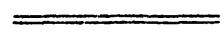

\section{Zur Frage der Quadriurate.}

(Bemerkungen zu der Abhandlung von Kohler ${ }^{1}$ ) über Komplexbildung in Lösungen von Harnsäure und harnsauren Salzen.)

Von

W. E. Ringer.

(Aus dem physiologischen Laboratorium der Universität Utrecht.)

(Der Redaktion zugegangen am 10. Jannar 1914.)

Rudolf Kohler hat sich in einigen Arbeiten²) mit der Frage der sogenannten Quadriurate beschäftigt und war dabei zu dem Schluß ge-

2) Diese Zeitschrift, Bd. 88, S. 259.

2) Ibidem, Bd. 70, S. 360; Bd. 72, S. 169. 